УДК 62-83:621.313

\title{
ОЦЕНКА ТЕПЛОВОГО СОСТОЯНИЯ ЭЛЕКТРОДВИГАТЕЛЕЙ ПЕРЕМЕННОГО ТОКА КОМПРЕССОРНЫХ СТАНЦИЙ МАГИСТРАЛЬНЫХ ГАЗОПРОВОДОВ
}

\author{
Зюзев Анатолий Михайлович1, \\ a.m.zyuzev@urfu.ru
}

\author{
Крюков Олег Викторович², \\ o.v.kryukov@mail.ru
}

\author{
Метельков Владимир Павлович1, \\ v.p.metelkov@urfu.ru
}

\author{
Михальченко Сергей Геннадьевич 3 , \\ msg@ie.tusur.ru \\ 1 Уральский федеральный университет, \\ Россия, 620002, г. Екатеринбург, ул. Мира, 19. \\ 2 OO० «ТСН-электро», \\ Россия, 603108, г. Нижний Новгород, ул. Электровозная, 7 «А». \\ 3 Томский университет систем управления и радиоэлектроники, \\ Россия, 634050, г. Томск, пр. Ленина, 40.
}

\begin{abstract}
Актуальность исследования обусловлена необходимостью оснащения системами мониторинга основного технологического оборудования компрессорных станций магистральных газопроводов. Эти системы должны обеспечивать достоверную оценку текущего технического состояния компрессорных станций и прогнозировать его динамику на интервале между измерениями. Инфоормации, получаемой с датчиков, не всегда достаточно для такого прогнозирования, что обуславливает необходимость использования подходов, основанных на моделировании.

Цель: выявить возможности мониторинга теплового состояния обмотки статора крупных электродвигателей переменного тока аналитическими методами и методами моделирования с использованием информации, полученной с датчиков температуры.

Объекты: двигатели переменного тока электроприводных газоперекачивающих агрегатов.

Методы: аналитические методы, а также моделирование с использованием термодинамических моделей электродвигателя на основе тепловых схем с сосредоточенными параметрами.

Результаты. Аналитическим путем получены соотношения, позволяющие выполнить приближенную оценку теплового состояния обмотки статора крупных электродвигателей переменного тока на основе трехмассовой тепловой схемы. Приведены результаты моделирования на основе тепловых схем с сосредоточенными параметрами нагрева обмотки статора крупного электродвигателя переменного тока с радиальными каналами охлаждения. Показано, что этот подход позволяет получить распределение температуры вдоль обмотки в аксиальном направлении, что дает возможность сравнительно точно оиенить тепловое состояние обмотки, избежав использования очень требовательных к вычислительным ресурсам подходов на основе метода конечных элементов и вычислительной потоковой динамики. Предложено использование остаточного термического ресурса обмотки статора для прогнозирования ориентировочного срока ее службы на основе информации о температуре обмотки, получаемой с термодатчиков или из термодинамической модели.
\end{abstract}

\section{Ключевые слова:}

Магистральный газопровод, газоперекачивающий агрегат, компрессорная станция, электродвигатели переменного тока, изоляция обмотки статора, термодинамическая модель, тепловая схема замещения с сосредоточенными параметрами, термический ресурс изоляции.

\section{Введение}

Общая протяженность газопроводов в Российской Федерации превышает 170 тыс. км. Это делает очевидной важность задачи обеспечения надежности и эффективности эксплуатации магистральных газопроводов (МГ) [1]. Одним из важнейших элементов в составе систем МГ, являются компрессорные станции (КС) [2], количество которых на территории России превышает четверть тысячи. Эти станции служат управляющими элементами, которые обеспечивают нужный режим функционирования газопровода, а надежность их оборудования в значительной степени влияет на надежность системы МГ в целом.
Совершенствование компьютерных технологий и прогресс в элементной базе систем автоматизации открывают новые возможности для снижения аварийности таких сложных систем, как КС МГ ПАО «Газпром» [3-5]. Задачей автоматизированных систем управления КС является повышение надежности, энергетической эффективности и безопасности функционирования МГ. В этом контексте также следует отметить и то, что все МГ Российской Федерации относятся к промышленным объектам повышенной опасности и требования к обеспечению надежности и безаварийности работы их оборудования, устанавливаемые федеральными и отраслевыми нормативно- 
техническими документами, с каждым годом становятся все более строгими [6-9].

Все эти обстоятельства заставляют уделять все большее внимание совершенствованию систем мониторинга состояния оборудования КС и создания автоматизированных систем диагностики с использованием современной вычислительной техники [9, 10]. Целый ряд отраслевых нормативных документов содержит прямые требования по оснащению системами мониторинга основного технологического оборудования КС $[6,11,12]$. Эти документы регламентируют реализацию мониторинга работоспособности, надежности и безопасности оборудования КС с помощью технических и программных средств, которые должны обеспечивать диагностику технического состояния оборудования, а также его прогнозирование на интервале времени между измерениями. Причем установка таких систем должна обеспечиваться как при строительстве новых КС, так и в процессе модернизации эксплуатируемых.

В ПАО «Газпром» (в частности, на КС ООО «Газпром трансгаз Нижний Новгород») в ходе модернизации систем автоматики электроприводных газоперекачивающих агрегатов (ЭГПА) были заменены старые релейные и аналоговые системы автоматического управления на микропроцессорные системы типа КРУГ-2000, САУ КАРАТ-М. Однако эти системы в основном позволяют реализовывать управление и контроль над процессами, происходящими лишь в механической и технологической части ЭГПА, и практически не позволяют обеспечить мониторинг технического состояния приводного высоковольтного электродвигателя ЭГПА. Рассмотрим некоторые вопросы, связанные с тепловым состоянием двигателей ЭГПА.

\section{Особенности тепловых процессов}

\section{в крупных электродвигателях переменного тока}

Важным фактором, влияющим на вероятность выхода двигателя из строя, является температура статорной обмотки, определяющая скорость термического старения изоляции. Для выяснения диапазона колебаний температуры элементов статора в процессе функционирования ЭГПА было проведено измерение температуры при пуске и останове приводного двигателя, а также при изменениях режима работы МГ. На рис. 1 показано, как изменяется температура элементов статора двигателя при пуске и после остановки ЭГПА.

Исследования теплового состояния двигателя ЭГПА, проводившиеся на компрессорных станциях «Починковская» и «Сеченовская», позволили установить, что в летний период колебания температуры статора в основном коррелируют с суточными колебаниями температуры окружающей среды. В зимний период колебания температуры наружного воздуха оказывают слабое влияние на температуру двигателя, в этом случае его тепловое состояние главным образом зависит от количества ЭГПА, одновременно работающих в КС. После останова ЭГПА в течение некоторого интервала времени происходит «тепловой удар» - подъ- ем температуры меди и стали статора на $10-15{ }^{\circ} \mathrm{C}$, в результате чего температура обмотки может превысить допустимые значения для соответствующего класса изоляции.
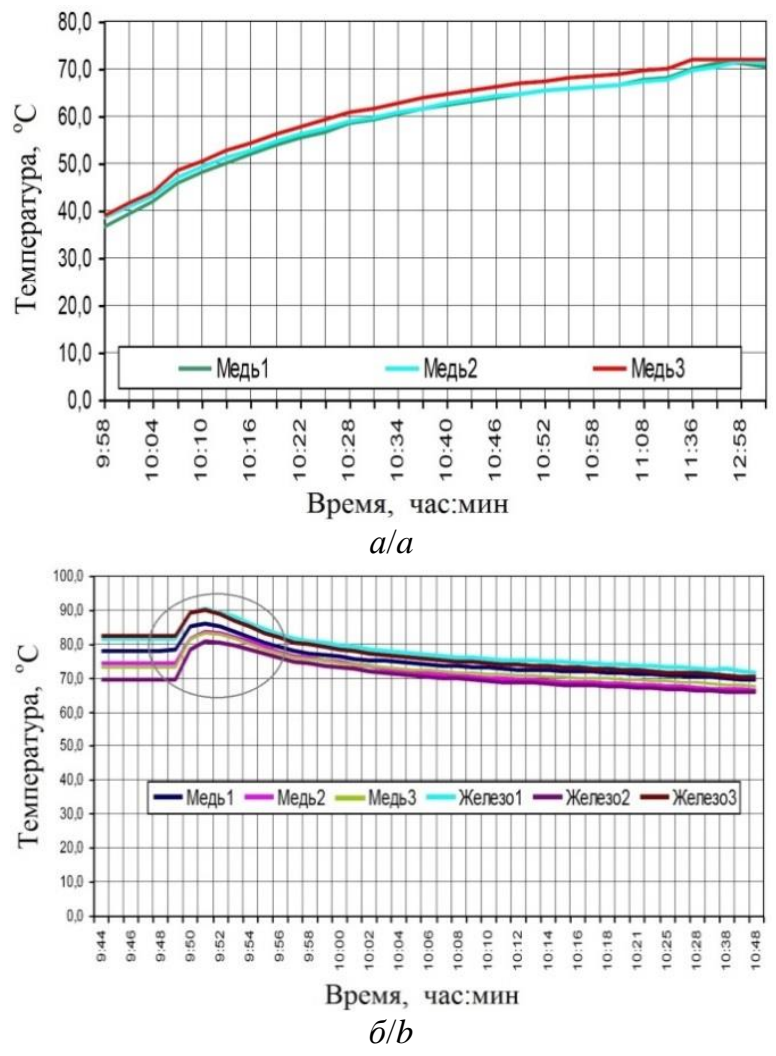

Pис. 1. Проиессы изменения температуры статора синхронного двигателя: а) после запуска ЭГПА № 6 компрессорного ичеха "Ямбург-Елеи-2» КС-25 «Починковская»; б) после останова ЭГПА № 1 компрессорного чеха «Ямбург-Елеи-1» КС-25 «Починковская» (овалом выделена область «теплового удара»)

Fig. 1. Synchronous motor stator temperature change: a) at start-up of Electrically-Driven Gas-Pumping Unit (EGPU) No. 6 at compressor manufactory «YamburgElets-2»KS-25 «Pochinkovskaya»; b) after stop of EGPU No. 1 at compressor manufactory «YamburgElets-1»KS-25 «Pochinkovskaya» (the oval marked the area of «heat stroke»)

Поскольку перегрев в данной области, хорошо различимой на графике (рис. 1, б), кратковременный, то наилучшим решением данной проблемы было бы повышение нагревостойкости изоляции, что позволило бы снизить скорость ее термического старения. Как правило, возможности запаса температурной устойчивости изоляции в электромоторе уже находятся на пределе возможного, в этом случае решением могла бы стать принудительная вентиляция ЭГПА. Но и такое решение не везде может быть применено, тогда остается уповать на точность расчета показателей старения и износа изоляции обмоток, что может быть реализовано только при наличии достаточно точной модели температурного состояния электропривода. 
При двухсторонней системе вентиляции электродвигателя (как, например, у СТД-12500-2), когда поток охлаждающего воздуха нагнетается в область пазовых частей обмотки, наибольшую температуру имеет ее средняя часть. Это хорошо прослеживается на рис. 2, где показана термограмма статора двигателя работающего ЭГПА № 7 компрессорного цеха «Ямбург-Западная граница» КС «Починковская», полученная с помощью тепловизора «Теrma CAM E25».

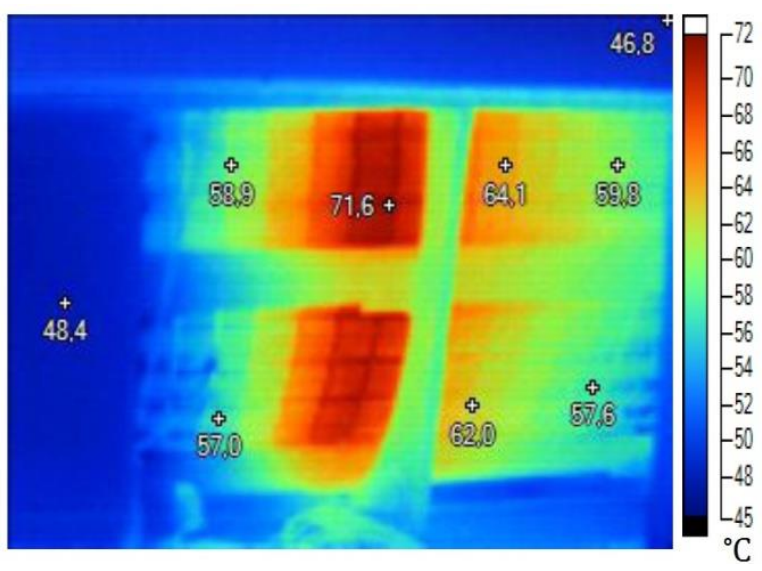

$a / a$

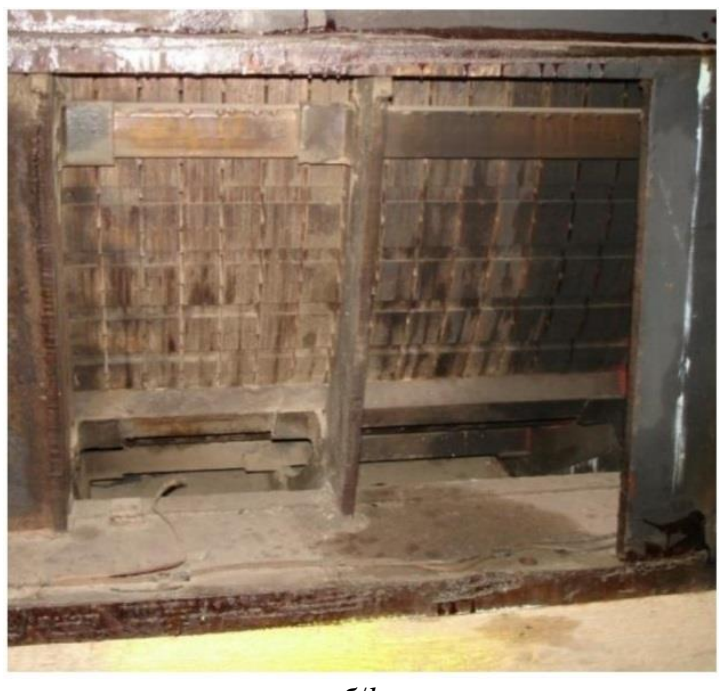

$\sigma / b$

Pис. 2. Статор работающего синхронного двигателя (ЭГПА № 7 МГ «Ямбург-Западная граница»): а) термограмма; б) фотография соответствующчей области статора

Fig. 2. Stator of an operating synchronous motor (EGPU No. 7 of the "Yamburg-Zapadnaya granitsa» trunk gas pipeline): a) thermogram; b) photo of the corresponding stator area

На термограмме видно, что температура средней части статора примерно на $20^{\circ} \mathrm{C}$ превышает температуру наиболее холодной области. Аналогичная картина наблюдается и на других электродвигателях ЭГПА. Соотнеся этот факт с тем, что, согласно статистическим данным, около 86 \% случаев пробоя изоляции приходится именно на пазовую часть обмотки статора (ближе к середине паза в аксиальном направлении), можно сделать вывод, что именно температу- ра оказывает наиболее существенное влияние на вероятность пробоя изоляции статорной обмотки приводного двигателя ЭГПА. Отсюда вытекает вывод о важности адекватной оценки теплового состояния обмотки статора этих двигателей.

Высоковольтные синхронные электродвигатели (СД), применяемые в ЭГПА, представляют собой достаточно сложный объект [13]. Можно построить для них модели, которые будут математически описывать их функционирование с различных сторон (тепловое, электромагнитное состояние, механические нагрузки и т. д.). Каждая такая модель может быть применена для прогноза технического состояния двигателя. Средства диагностирования могут быть встроенными в сам объект мониторинга, что позволяет использовать существующие или вновь вводимые датчики электромагнитных и механических величин, характеризующих работу двигателя, а модель состояния исправного двигателя может быть легко реализована с помощью вычислительных средств. Как правило, набор основных параметров, характеризующих состояние электродвигателя начинает изменяться еще до наступления отказа. В этом случае ЭГПА может быть остановлен еще до возникновения аварии. Что важно, при этом можно получить и предысторию развития процесса изменения состояния двигателя.

Природа физических процессов, обуславливающих проявление деградации статорной изоляции, является различной, здесь рассмотрим возможности учета влияния на изоляцию термических факторов в системе мониторинга состояния двигателя ЭГПА.

\section{Нагрев обмотки статора электродвигателя ЭГПА}

В настоящее время температура меди и стали статора двигателя ЭГПА контролируется штатными температурными датчиками типа ТСМ-50 (ТСМ-9502). Эти датчики размещаются по три в каждой фазе (рис. 3). Те, которые на дне паза, контролируют температуру «стали», а датчики между слоями обмотки температуру «меди». Практика эксплуатации ЭГПА свидетельствует о том, что такого набора датчиков слишком мало для получения полной информации о температурном состоянии статора, поскольку соотношение температуры элементов статора и ее пространственное распределение изменяется при изменении режимов работы.

Необходимо иметь в виду, что подробность и точность модели, применяемой для задач диагностирования, всегда определяет глубину и достоверность результатов диагностики состояния объекта. Для мониторинга и прогнозирования температурных режимов изоляции обмотки статора возможно использование одной из тепловых моделей нагрева двигателей переменного тока [14]. В инженерной практике для оценки теплового состояния электродвигателей широкое распространение получила так называемая «одномассовая модель нагрева», в которой электродвигатель рассматривается как однородное тело с единственным источником греющих потерь. Однако такая модель для мощных машин с большими габаритами и существенной неравномерностью пространственного 
распределения температуры не обеспечивает адекватные результаты с точки зрения оперативного мониторинга теплового состояния обмотки статора.

Наиболее точные результаты при моделировании теплового состояния двигателя могут быть получены с использованием метода конечных элементов (Finite Element Method - FEM) [15-18] или методов вычислительной потоковой динамики (Computational Fluid Dynamic - CFD) [19-22]. Однако эти методы предъявляют очень высокие требования к вычислительным ресурсам. Это вытекает из того факта, что термодинамические задачи, в отличие от электродинамических, как правило, требуют 3D-моделей. Поэтому такие подходы сложно применять для онлайн мониторинга теплового состояния электрической машины [23-25].

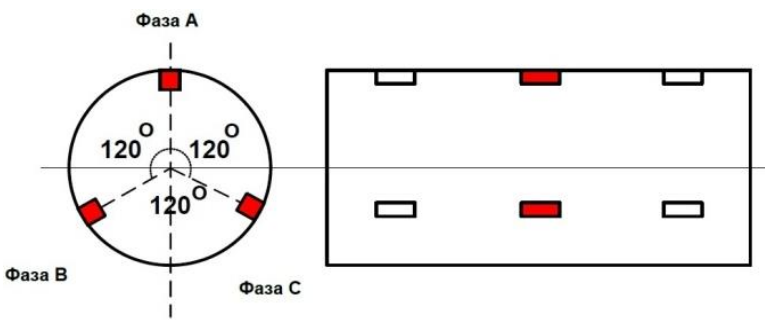

$\square$ Штатные датчики «меди» в обмотке «Монолит-2»

$\square$ Доп. датчики «меди» в гибкой многослойной обмотке $a / a$

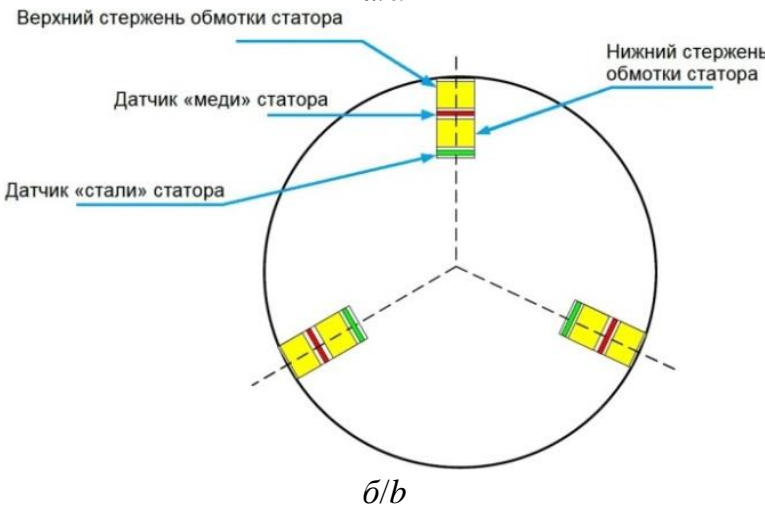

Рис. 3. Расположение датчиков температуры «меди» $и$ «стали» в статоре электродвигателя ЭГПА а) схема расположения датчиков температуры TCM-50; б) конструкиия обмоток статора с датчиками

Fig. 3. Location of temperature sensors of "copper» and "steel» in the stator of the EGPU electric motor: a) layout of the temperature sensors TSM-50, b) design of stator windings with sensors

Термодинамические модели на базе тепловых схем замещения с сосредоточенными параметрами для этих целей значительно более применимы, поскольку обеспечивают гораздо более высокую скорость расчета при меньших требованиях к вычислительным возможностям [26-28]. Существуют достаточно подробные термодинамические модели крупных машин переменного тока такого типа. Так, в $[29,30]$ представлена термодинамическая модель двигателя АДЧР-1600 (1,6 МВТ), тепловая схема ко- торой содержит 71 узел. Эта модель позволяет визуализировать распределение температуры обмотки статора в аксиальном направлении (рис. 4), обеспечивая при этом весьма небольшое время расчета (порядка одной секунды времени расчета на одну секунду модельного времени). Превышение температуры обмотки статора в средней части паза при работе с номинальной нагрузкой соответствует допустимому по ГОСТ превышению температуры обмотки для изоляции класса F. Обратим внимание, что распределение температуры вдоль обмотки на рис. 4 хорошо согласуется с распределением температуры на рис. 2.

Кратко остановимся на возможностях использования аналитического подхода в задачах оценки теплового состояния электродвигателя ЭГПА. В векторноматричной форме термодинамическая модель электрической машины может быть представлена в виде уравнения

$$
\boldsymbol{C} \frac{d \boldsymbol{\theta}}{d t}=\Delta \boldsymbol{P}+\Lambda \boldsymbol{\theta}
$$

где $\boldsymbol{\theta}$ и $\boldsymbol{\Delta} \boldsymbol{P}$ - векторы температур узлов модели и мощности потерь в этих узлах; $\boldsymbol{C}$ - диагональная матрица теплоемкостей узлов; $\Lambda$ - матрица тепловых проводимостей.

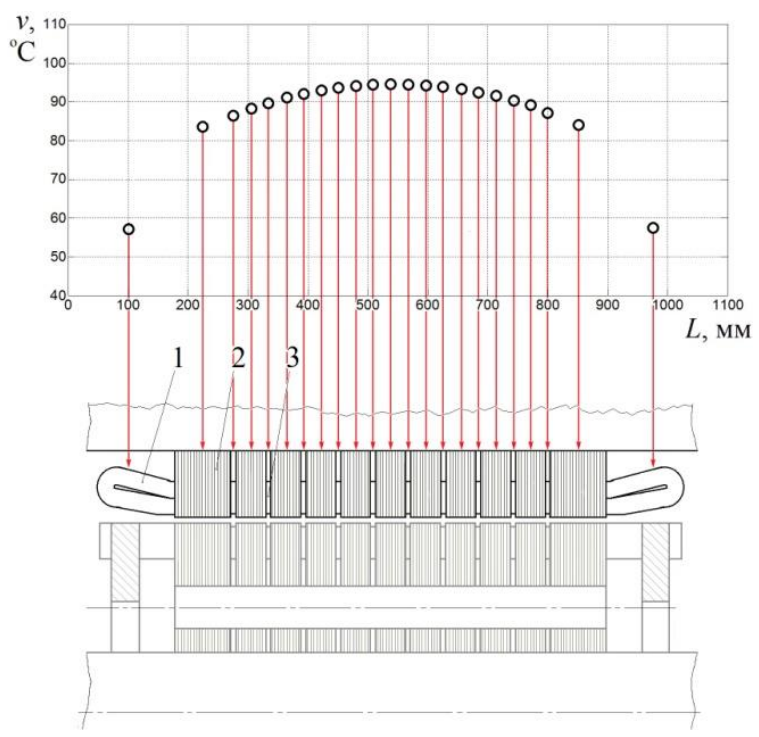

Рис. 4. Превышение температуры обмотки статора двигателя АДЧР-1600 в установившемся режиме при номинальной нагрузке (1 - лобовые части обмотки статора; 2 - сталь статора; 3 - пазовая часть обмотки статора; серым иветом изображены элементы ротора)

Fig. 4. Temperature rise of the ADChR-1600 motor stator winding in steady state at rated load (1 - stator winding end parts; 2 - stator steel; 3 - stator winding slotted part; rotor elements are shown in gray)

Модель (1) предполагает в общем случае использование вычислительной техники и наличие достаточно подробной информации о внутренних параметpax и конструктивных особенностях машины. Поэтому в ряде случаев может быть полезен максимально 
упрощенный вариант модели, дающий возможность аналитического исследования. Для получения аналитическим путем некоторых общих выводов об особенностях нагрева синхронного двигателя ЭГПА редуцируем модель (1) до модели третьего порядка. В этом случае приходим к термодинамической модели СД в виде трёх элементов (рис. 5): меди обмотки статора, стали самого статора и корпуса, а также ротора вместе с обмоткой возбуждения, сталью сердечника, валом, подшипниками. На рис. 5 изображена модель СД, где $v_{1}, v_{2}, v_{3}$ - превышения температур обмотки статора, стали статора и ротора над температурой окружающей среды; $C_{1}, C_{2}, C_{3}$ - теплоёмкости обмотки статора, стали статора и ротора; $\Lambda_{1}, \Lambda_{2}, \Lambda_{3}$ - коэффициенты теплоотдачи соответствующих элементов СД в окружающую среду; $\Lambda_{31}, \Lambda_{21}$ - коэффициенты теплопередачи между греющимися элементами модели.

Уравнения теплового баланса для такой термодинамической модели третьего порядка выглядят следующим образом:

$$
\left.\begin{array}{l}
C_{1} \frac{d v_{1}}{d t}+\Lambda_{1} v_{1}+\Lambda_{12}\left(v_{1}-v_{2}\right)+\Lambda_{13}\left(v_{1}-v_{3}\right)=\Delta P_{M 1} \\
C_{2} \frac{d v_{1}}{d t}+\Lambda_{2} v_{2}+\Lambda_{21}\left(v_{2}-v_{1}\right)+\Lambda_{23}\left(v_{2}-v_{3}\right)=\Delta P_{C 1} \\
C_{3} \frac{d v_{3}}{d t}+\Lambda_{3} v_{3}+\Lambda_{31}\left(v_{3}-v_{1}\right)+\Lambda_{32}\left(v_{3}-v_{2}\right)=\Delta P_{2},
\end{array}\right\}
$$

где $\Lambda_{12}, \Lambda_{21}$ - тепловые проводимости между медью и сталью статора через пазовую изоляцию; $\Lambda_{13}, \Lambda_{31}-$ тепловые проводимости между обмоткой статора и ротором через пазовую изоляцию и воздушный зазор; $\Lambda_{23}, \Lambda_{32}$ - тепловые проводимости между сталью статора и ротором через воздушный зазор; $\Delta P_{M 1}, \Delta P_{\mathrm{Cl}}$, $\Delta P_{2}$ - мощности потерь соответственно в обмотке статора, стали статора и суммарные потери в роторе.

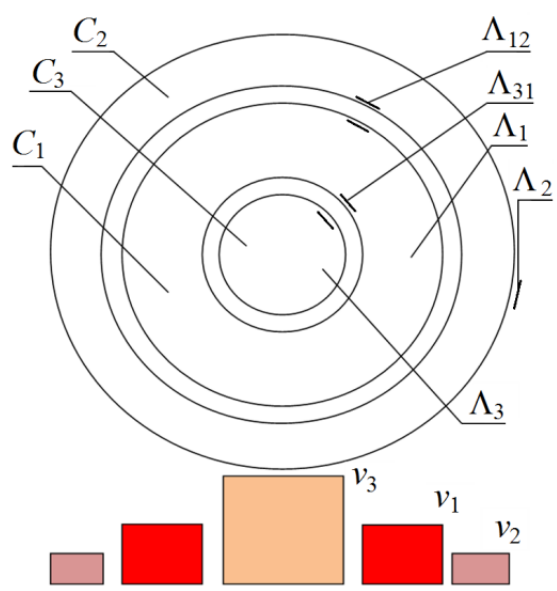

Рис. 5. Термодинамическая модель третьего порядка для синхронного двигателя

Fig. 5. Third-order thermodynamic model for a synchronous motor

В установившемся режиме, когда температура не изменяется, получим

$$
\frac{d v_{1}}{d t}=\frac{d v_{2}}{d t}=\frac{d v_{3}}{d t}=0
$$

С учетом (3) система дифференциальных уравнений (2) вырождается в систему алгебраических уравнений, которая, с учетом температурной зависимости мощности потерь в обмотке статора, может быть записана в виде векторно-матричного уравнения

$$
\left\|\begin{array}{ccc}
a_{1} & -\Lambda_{12} & -\Lambda_{13} \\
-\Lambda_{21} & a_{2} & -\Lambda_{23} \\
-\Lambda_{31} & -\Lambda_{32} & a_{3}
\end{array}\right\| \times\left\|\begin{array}{l}
v_{1} \\
v_{2} \\
v_{3}
\end{array}\right\|=\left\|\begin{array}{l}
\Delta P_{M 10} \\
\Delta P_{C 1} \\
\Delta P_{2}
\end{array}\right\|,
$$

где

$$
\left.\begin{array}{l}
a_{1}=\Lambda_{1}+\Lambda_{12}+\Lambda_{13}-\alpha I_{1 N}^{2} R_{1} ; \\
a_{2}=\Lambda_{2}+\Lambda_{21}+\Lambda_{23} ; \\
a_{3}=\Lambda_{3}+\Lambda_{31}+\Lambda_{32} .
\end{array}\right\}
$$

В выражениях (4) и (5) обозначено: $\Delta P_{M 10}-$ мощность потерь в обмотке статора в начале нагрева; $\alpha-$ температурный коэффициент меди; $I_{1 N}-$ номинальный ток статора; $R_{1}$ - сопротивление обмотки статора.

Из системы (4), принимая во внимание выражения (5), можем найти установившиеся значения превышений температуры $v$ :

$$
\left.\begin{array}{l}
v_{1}=\frac{1}{a_{1}}\left(\Delta P_{M 10}+\Lambda_{12} \frac{b_{2}}{b_{1}}+\Lambda_{13} \frac{b_{4}}{b_{3}}\right) ; \\
v_{2}=b_{2} / b_{1} ; \\
v_{3}=b_{4} / b_{3},
\end{array}\right\}
$$

где

$$
\begin{gathered}
b_{1}=\frac{a_{1} a_{2}-\Lambda_{21} \Lambda_{12}}{\Lambda_{21} \Lambda_{13}-\Lambda_{23} a_{1}}-\frac{a_{1} \Lambda_{32}+\Lambda_{31} \Lambda_{12}}{a_{1} a_{3}-\Lambda_{31} \Lambda_{13}} ; \\
b_{2}=\frac{a_{1} \Delta P_{C 1}+\Lambda_{21} \Delta P_{M 10}}{\Lambda_{21} \Lambda_{13}-\Lambda_{23} a_{1}}+\frac{a_{1} \Delta P_{2}+\Lambda_{31} \Delta P_{M 10}}{a_{1} a_{3}-\Lambda_{31} \Lambda_{13}} ; \\
b_{3}=-\frac{a_{1} a_{3}-\Lambda_{31} \Lambda_{13}}{\Lambda_{31} \Lambda_{12}+\Lambda_{32} a_{1}}-\frac{\Lambda_{21} \Lambda_{13}-\Lambda_{23} a_{1}}{a_{1} a_{2}-\Lambda_{21} \Lambda_{12}} ; \\
b_{4}=-\frac{a_{1} \Delta P_{2}+\Lambda_{31} \Delta P_{M 10}}{\Lambda_{31} \Lambda_{12}+\Lambda_{32} a_{1}}-\frac{a_{1} \Delta P_{C 1}+\Lambda_{21} \Delta P_{M 10}}{a_{1} a_{2}-\Lambda_{21} \Lambda_{12}} .
\end{gathered}
$$

Анализ результатов, полученных с помощью термодинамической модели третьего порядка, показал, что для двигателя СТД-12500-2:

- превышение температуры стали статора $v_{2}$ хорошо коррелирует с превышением температуры обмотки статора $v_{1}$, а превышение температуры ротора $v_{3}$ существенно отличается как от $v_{1}$, так и от $v_{2}$. Это объясняется соотношением величин тепловых проводимостей, где $\Lambda_{12}>>\Lambda_{23}>\Lambda_{13}$;

- превышение температуры обмотки статора СД, полученное с помощью модели (4)-(6), для номинальной нагрузки на валу близко к стандартной рабочей температуре, установленной ГОСТ для обмоток с изоляцией соответствующего класса нагревостойкости.

\section{Оценка ресурса и срока службы изоляции обмоток}

Информация с датчиков температуры обмотки или термодинамическая модель машины позволяет выполнить оценку остаточного термического ресурса 
изоляции обмотки и прогнозировать ориентировочный срок ее службы.

Срок службы изоляции обмотки статора при температуре $\theta\left({ }^{\circ} \mathrm{C}\right)$ связан с температурой экспоненциальной зависимостью:

$$
T=A e^{-\gamma \theta},
$$

где $A, \gamma$ - постоянные, зависящие от класса нагревостойкости изоляции.

Расход термического ресурса изоляции за время $t_{\mathrm{p}}$ можно представить как

$$
\Delta R_{t}=\int_{0}^{t_{\mathrm{p}}} \xi(t) d t,
$$

где $\xi$ - скорость термического старения изоляции (величина, обратная $T$, при этом значении $\theta$ ).

Тогда остаточный ресурс изоляции обмотки можно определить как $R_{r e s}=1-\Delta R_{t}$, где $\Delta R_{t}$ рассчитывается по выражению (8) с учетом зависимости (7). Если имеется возможность вычислять или измерять температуру в нескольких точках обмотки, величина остаточного термического ресурса, как и температура, будет векторной величиной. В таком случае минимальная компонента этого вектора и будет определять значение остаточного термического ресурса обмотки.

Следует также упомянуть другое перспективное направление развития систем мониторинга состояния изоляции обмотки статора электрических машин, касающееся возможности построения системы мониторинга на основе емкостных токов утечки, генерируемых фронтами приложенного к обмотке напряжения. В работе [31] показана тесная связь между амплитудой емкостных токов утечки $\left(I_{\mathrm{y}}\right)$ и процессом термической деградации свойств изоляции обмотки. Эта зависимость может быть описана убывающей функцией вида

$$
I_{\mathrm{y}}=\Delta I_{\mathrm{y} \max } e^{-\alpha_{\mathrm{y}} t}+I_{\mathrm{y} 0},
$$

где $\Delta I_{\mathrm{y} . \max }, \alpha_{\mathrm{y}}$ и $I_{\mathrm{y} 0}-$ постоянные; $t$ - наработка машины.

\section{СПИСОК ЛИТЕРАТУРЫ}

1. Пужайло А.Ф. Энергоснабжение и автоматизация энергооборудования компрессорных станций. Т. 2. - Н-Новгород: Вектор ТиС, 2011. - 664 с

2. Козаченко А.Н. Эксплуатация компрессорных станций. - М.: Нефть и газ, 1999. - 463 с.

3. Репин Д.Г., Крюков О.В. Концепты системы мониторинга технического состояния компрессорных станций // Контроль. Диагностика. - 2017. - № 12. - С. 30-35.

4. Kryukov O.V., Serebryakov A.V. Energy efficient power supply systems of oil and gas pipelines electric drives // Вестник ЮУрГУ. Серия: Энергетика. - 2017. - Т. 17. - № 3. - С. 102-110.

5. Kryukov O.V. Scientific background for the development of intelligent electric drives for oil and gas process units // Вестник ЮУрГУ. Энергетика. - 2017. - Т. 17. - № 1. - С. 56-62.

6. Правила эксплуатации магистральных газопроводов // СТО Газпром 2-3.5-454-2010. - М.: ОАО «Газпром», 2010. - 216 с.

7. Применение и перспективы развития электропривода ГПА на объектах реконструкции и нового строительства ОАО «Газпром». Отчет о НИР в 3-х т. - Нижний Новгород: ОАО «Гипрогазцентр», 2012.
На основе зависимости (9) появляется возможность мониторинга текущего состояния изоляции, а главное - оценки ее остаточного ресурса $[32,33]$. Поскольку амплитуда емкостных токов при увеличении наработки уменьшается по экспоненциальному закону, можно спрогнозировать значение наработки, при которой величина емкостных токов уйдет ниже некоторого порогового уровня, что и будет свидетельствовать об исчерпании ресурса изоляции. Таким образом становится возможным создание системы мониторинга и прогнозирования состояния изоляции обмотки статора, которая будет представлять одну из подсистем в рамках комплексной диагностической модели системы контроля технического состояния электродвигателя ЭГПА.

\section{Заключение}

Практика эксплуатации электроприводных газоперекачивающих агрегатов показывает, что обычно используемого количества датчиков температуры недостаточно для получения полной информации о температурном состоянии обмотки статора. Аналитические выражения на основе термодинамической модели третьего порядка позволяют получить приближенную оценку средней температуры обмотки статора, но для построения системы мониторинга теплового состояния машины требуется моделирование на основе метода тепловых схем. Такой подход позволяет учесть распределение температуры вдоль обмотки и другие особенности нагрева, связанные с учетом способа охлаждения, конфигурацией внешней поверхности машины, теплоотводом через станину, подшипниковые щиты и так далее. Термодинамические модели могут быть верифицированы с использованием информации, полученной с датчиков температуры. Подобные модели, в сочетании с мониторингом состояния изоляции на основе регистрации емкостных токов утечки, позволяют обеспечить оценку остаточного ресурса изоляции обмотки и прогноз ориентировочного срока ее службы.

8. Программа повышения надежности работы и эффективности компрессорных станций с ЭГПА. - М.: ООО «ВНИИГАЗ», 2002. - $102 \mathrm{c}$

9. Аксютин О.Е. Современное состояние газотранспортной системы России // Газовая промышленность. - 2010. - № 10. C. $8-11$.

10. Проблемы мощных электрических машин на сессии СИГРЭ2008 / Б.А. Алексеев, Ф.А. Поляков, Л.Г. Мамиконянц, Ю.Г. Шакаран // Электричество. - 2009. - № 3. - С. 60-67.

11. Крюков О.В. Оценка эксплуатационных факторов ЭГПА по нормативным требованиям мониторинга // Контроль. Диагностика. - 2018. - № 10. - С. 50-57.

12. Правила технической эксплуатации магистральных газопроводов. Ведомственный руководящий документ ВРД 39-1.10006-2000. - М.: ИРЦ Газпром, 2002. - 147 с.

13. Копылов И.П. Математическое моделирование электрических машин. - М.: Высшая школа, 1994. - 318 с.

14. Крюков О.В. Моделирование термодинамических процессов в системах с мощными электрическими машинами // Приборы и системы. Управление, контроль, диагностика. - 2018. - № 9. C. 9-15.

15. De Santiago Ochoa J. FEM analysis applied to electric machines for electric vehicles. PhD Diss. Abstract. - Uppsala, 2011. - 54 p. 
16. Anandkumar S Malipatil, Ravinder Reddy P. Thermal stress analysis of DC motor using finite element method // International Journal of Engineering Research \& Technology (IJERT). 2014. - V. 3. - Iss. 9. - P. 283-290.

17. Numerical study on electromagnetics and thermal cooling of a switched reluctance motor / Jer-Huan Jang, Han-Chieh Chiu, WeiMonYan, M.C. Tsai, Pin-Yuan Wang // Case Studies in Thermal Engineering. - 2015. - V. 6. - P. 16-27.

18. Plejić M., Goričan V., Hribernik B. FEM thermal modeling of an induction motor // Electric and magnetic fields / Eds. A. Nicolet, R. Belmans. - Boston, MA: Springer, 1995. - P. 155-158.

19. CFD analyses of a radial fan for electric motor cooling / E. Galloni, P. Parisi, F. Marignetti, G. Volpe // Thermal Science and Engineering Progress. - 2018. - V. 8. - P. 470-476.

20. CFD study of forced air cooling and windage losses in a high speed electric motor / K.R. Anderson, Jun Lin, Ch. McNamara, V. Magri // Journal of Electronics Cooling and Thermal Control. 2015. - V. 5. - № 2. - P. 27-44.

21. Borges S.S., Cezario C.A., Kunz T.T. Design of water cooled electric motors using CFD and thermography techniques // Proc. 2008 18th International Conference on Electrical Machines. Vilamoura, Portugal, 6-9 September 2008. - P. 1-6.

22. Pechánek R., Bouzek L. Analyzing of two types water cooling electric motors using computational fluid dynamics // Conf. Paper of 15th International Power Electronics and Motion Contro Conference, EPE-PEMC 2012 ECCE Europe. - Novi Sad, Serbia, 4-6 September, 2012. - P. 1-5.

23. Boglietti A., Cavagnino A., Staton D.A. TEFC induction motor thermal models: a parameter sensitivity analysis // IEEE Transactions on Industry Applications. - 2005. - V. 41. - Iss. 3. P. 756-763

24. Chin Y.K., Staton D.A. Transient thermal analysis using both lumped-circuit approach and finite element method of a permanent magnet traction motor // Proc. IEEE Africon. - Gaborone, Botswana, 2004. - P. 1027-1035.

25. Thermal modelling of an alternator for use in a prediction system / J. Graham, R. Dixon, K. Gregory, J. Pearson // Proc. 2012
UKACC International Conference on Control. - Cardiff, UK, 3-5 September, 2012. - P. 455-460.

26. Evolution and modern approaches for thermal analysis of electrical machines / A. Boglietti, A. Cavagnino, D. Staton, M. Shanel, M. Mueller, C. Mejuto // IEEE Transactions On Industrial Electronics. - 2009. - V. 56. - Iss. 3. - P. 871-882

27. Krok R. Electric mine motor thermal models aiding design and setting thermal protections // Bulletin of the Polish Academy of Sciences. Technical Sciences. - 2012. - V. 60. - № 1. - P. 103-110.

28. Experimental and CFD investigation of a lumped parameter thermal model of a single-sided, slotted axial flux generator / C.H. Lim, G. Airoldi, J.R. Bumby, R.G. Dominy, G.I. Ingram, K. Mahkamov, N.L. Brown, A. Mebarki, M. Shanel // International Journal of Thermal Sciences. - 2010. - V. 49. - Iss. 9. P. 1732-1741.

29. Ziuzev A.M., Metelkov V.P. Research of the start-up modes of multi-stage blower asynchronous drive // Proc. IEEE IX Int. Conf. on Power Drives Systems (ICPDS). - Perm, Russia, October 3-7, 2016. - P. 1-5

30. Ziuzev A.M., Metelkov V.P. On an estimate of the rotor winding temperature at start-up of high-voltage induction motor // Proc. 2017 International Conference on Industrial Engineering, Applications and Manufacturing (ICIEAM 2017). - SaintPetersburg, Russia, 16-19 May, 2017. - P. 1-6.

31. On-line capacitance and dissipation factor monitoring of $\mathrm{AC}$ stator insulation / K. Younsi, P. Neti, M. Shah, J. Zhou, J. Krahn, K. Weeber, C. Whitefield // IEEE Transactions on Dielectrics and Electrical Insulation. - 2010. - V. 17. - Iss. 5. - P. 1441-1452.

32. О возможности использования токов утечки для оценки состояния изоляции асинхронного двигателя / В.П. Метельков, М.Ю. Бородин, К.А. Кондаков, К.Е. Нестеров // Вестник ЮУрГУ. Серия: Энергетика. - 2019. - Т. 19. - № 1. - С. 67-74.

33. Метельков В.П., Зюзев А.М., Черных И.В. Система оценки остаточного ресурса изоляции обмотки асинхронного двигателя на основе емкостных токов утечки // Электротехнические системы и комплексы. - 2019. - № 1 (42). - С. 53-58.

Поступила 23.12.2020 2.

\section{Информация об авторах}

Зюзев A.M., доктор технических наук, профессор кафедры электропривода и автоматизации промышленных установок Уральского федерального университета.

Крюков О.В., доктор технических наук, заместитель директора по науке ООО «ТСН-электро».

Метельков В.П., доктор технических наук, профессор кафедры электропривода и автоматизации промышленных установок Уральского федерального университета.

Михальченко $\boldsymbol{C . \Gamma . , ~ д о к т о р ~ т е х н и ч е с к и х ~ н а у к , ~ з а в е д у ю щ и и ̆ ~ к а ф е д р о и ̆ ~ п р о м ы ш л е н н о и ̆ ~ э л е к т р о н и к и ~ Т о м с к о г о ~}$ университета систем управления и радиоэлектроники. 
UDC 62-83:621.313

\title{
ESTIMATION OF THERMAL STATE OF AC ELECTRIC MOTORS OF TRUNK GAS PIPELINES COMPRESSOR STATIONS
}

\author{
Anatoliy M. Ziuzev', \\ a.m.zyuzev@urfu.ru
}

\section{Oleg V. Kryukov²,} o.v.kryukov@mail.ru

Vladimir P. Metelkov', v.p.metelkov@urfu.ru

\author{
Sergey G. Mikhalchenko ${ }^{3}$, \\ msg@ie.tusur.ru \\ 1 Ural Federal University, \\ 19, Mira street, Ekaterinburg, 620002, Russia. \\ 2 TSN-electro LLC, \\ 7 A, Electrovoznaya street, Nizhniy Novgorod, 603108, Russia. \\ 3 Tomsk State University of Control Systems and Radioelectronics, \\ 40, Lenin avenue, Tomsk, 634050, Russia.
}

The relevance of the research is caused by the requirements for equipping the main technological equipment of compressor stations of trunk gas pipelines with monitoring systems. These systems should provide a reliable assessment of the current technical condition of the compressor station and predict its dynamics in the interval between measurements. The information received from the sensors is not always sufficient for such prediction, which necessitates the use of modeling-based approaches.

The main aim of the research is to reveal the possibilities of monitoring the thermal state of large AC motors stator winding by analytical methods and modeling methods using information obtained from temperature sensors.

Objects: AC motors of electric drive gas compressor units.

Methods: analytical methods, as well as simulation using thermodynamic models of an electric motor based on thermal circuits with lumped parameters.

Results. The authors have obtained analytically the relations that allow an approximate assessment of large AC motors stator winding thermal state based on a three-mass thermal circuit. The paper introduces the results of modeling based on thermal circuits with lumped parameters of heating the stator winding of a large AC electric motor with radial cooling channels. It is shown that this approach allows one to obtain the temperature distribution along the winding in the axial direction, which makes it possible to relatively accurately estimate the thermal state of the winding, avoiding the use of very computationally demanding approaches based on the finite element method and computational flow dynamics. It is proposed to use the residual thermal resource of the stator winding to predict its approximate service life based on information about the winding temperature obtained from temperature sensors or from a thermodynamic model.

\section{Key words:}

Trunk gas pipeline, gas pumping units, compressor station, AC electric motors, stator winding insulation, thermodynamic model, thermal circuits with lumped parameters, thermal insulation resource.

\section{REFERENCES}

1. Puzhaylo A.F. Energosberezhenie $i$ avtomatizatsiya elektrooborudovaniya kompressornykh stantsiy [Energy-saving electrical equipment and automation of compressor stations]. Nizhny Novgorod, Vektor TiS Publ., 2011. Vol. 2, 664 p.

2. Kozachenko A.N. Ekspluatatsiya kompressornykh stantsiy [Compressor station operation]. Moscow, Neft i gaz Publ., 1999. $463 \mathrm{p}$.

3. Repin D.G., Kryukov O.V. Kontsepty sistemy monitoringa tekhnicheskogo sostoyaniya kompressornykh stantsiy [Concepts of a monitoring system for the technical condition of compressor stations]. Kontrol. Diagnostika, 2017, no. 12, pp. 30-35.

4. Kryukov O.V., Serebryakov A.V. Energy efficient power supply systems of oil and gas pipelines electric drives. Bulletin of the South Ural State University. Ser. Power Engineering, 2017, vol. 17, no. 3, pp. 102-110. In Rus.

5. Kryukov O.V. Scientific background for the development of intelligent electric drives for oil and gas process units. Bulletin of the South Ural State University. Ser. Power Engineering, 2017, vol. 17, no. 1, pp. 56-62. In Rus.
6. Pravila ekspluatatsii magistralnykh gazoprovodov. STO Gazprom 2-3.5-454-2010 [Rules for the operation of trunk gas pipelines. STO Gazprom 2-3.5-454-2010]. Moscow, JSC Gazprom, 2010. $216 \mathrm{p}$.

7. Primenenie $i$ perspektivy razvitiya elektroprivoda GPA na obyektakh rekonstruktsii $i$ novogo stroitelstva OAO Gazprom [Application and development prospects of the GPU electric drive at the facilities of reconstruction and new construction of JSC Gazprom]. Report on research work in 3 vols. Moscow, JSC Giprogaztsentr, 2012. 327 p.

8. Programma povysheniya nadezhnosti raboty $i$ effektivnosti kompressornykh stantsiy s EGPA [The program of increasing operational reliability and efficiency of compressor stations with EGPU]. Moscow, VNIIGAZ LLC, 2002. 102 p.

9. Aksyutin O.E. Sovremennoe sostoyanie gazotransportnoy sistemy Rossii [The current state of the gas transmission system in Russia]. Gazovaya promyshlennost, 2010, no. 10. pp. 8-11.

10. Alekseyev B.A., Polyakov F.A., Mamikonyants L.G., Shakaran Yu.G. Problems of powerful electric machines at the CIGRE-2008 session. Elektrichestvo, 2009, no. 3, pp. 60-67. In Rus.

11. Kryukov O.V. Otsenka ekspluatatsionnykh faktorov EGPA po normativnym trebovaniyam monitoringa [Evaluation of 
operational factors of EGPU according to regulatory monitoring requirements]. Kontrol'. Diagnostika, 2018, no. 10, pp. 50-57.

12. Pravila tekhnicheskoy ekspluatatsii magistralnykh gazoprovodov. Vedomstvenny rukovodyashchiy dokument VRD 39-1.10-006-2000 [Operation Requirements for Trunk Gas Pipeline. Departmental guidance document VRD 39-1.10-006-2000]. Moscow, 2002.

13. Kopylov I.P. Matematicheskoe modelirovanie elektricheskikh mashin [Mathematical modeling of electrical machines]. Moscow, Vysshaya shkola Publ., 1994. 318 p.

14. Kryukov O.V. Modelirovanie termodinamicheskikh protsessov v sistemakh s moshchnymi elektricheskimi mashinami [Modeling thermodynamic processes in systems with powerful electric machines]. Instruments and Systems: Monitoring, Control, and Diagnostics, 2018, no. 9, pp. 9-15.

15. De Santiago Ochoa J. FEM analysis applied to electric machines for electric vehicles. PhD Diss. Abstract. Uppsala, 2011, $54 \mathrm{p}$.

16. Anandkumar S Malipatil, Ravinder Reddy P. Thermal Stress Analysis of DC Motor using Finite Element Method. International Journal of Engineering Research \& Technology (IJERT), 2014, vol. 3, Iss. 9, pp. 283-290.

17. Jer-Huan Jang, Han-Chieh Chiu, Wei-MonYan, Tsai M.C., PinYuan Wang. Numerical study on electromagnetics and thermal cooling of a switched reluctance motor. Case Studies in Thermal Engineering, 2015, vol. 6, pp. 16-27.

18. Plejić M., Goričan V., Hribernik B. FEM thermal modeling of an induction motor. Electric and Magnetic Fields. Eds. A. Nicolet, R. Belmans. Boston, MA, Springer, 1995. pp. 155-158.

19. Galloni E., Parisi P., Marignetti F., Volpe G. CFD analyses of a radial fan for electric motor cooling. Thermal Science and Engineering Progress, 2018, vol. 8, pp. 470-476.

20. Anderson K.R., Jun Lin, McNamara Ch., Magri V. CFD study of forced air cooling and windage losses in a high speed electric motor Journal of Electronics Cooling and Thermal Control, 2015, vol. 5, no. 2, pp. 27-44.

21. Borges S.S., Cezario C.A., Kunz T.T. Design of water cooled electric motors using CFD and thermography techniques, Proc. $200818^{\text {th }}$ International Conference on Electrical Machines. Vilamoura, Portugal, 6-9 September 2008. pp. 1-6.

22. Pechánek R., Bouzek L. Analyzing of two types water cooling electric motors using computational fluid dynamics. Paper of $15^{\text {th }}$ International Power Electronics and Motion Control Conference, EPE-PEMC 2012 ECCE Europe. Novi Sad, Serbia, 4-6 September 2012. pp. 1-5.

23. Boglietti A., Cavagnino A., Staton D.A. TEFC induction motors thermal models: A parameter sensitivity analysis. IEEE
Transactions on Industry Applications, 2005, vol. 41, Iss. 3, pp. 756-763.

24. Chin Y.K., Staton D.A. Transient thermal analysis using both lumped-circuit approach and finite element method of a permanent magnet traction motor. Proc. IEEE Africon. Gaborone, Botswana, 5-17 September 2004. pp. 1027-1035.

25. James Graham, Roger Dixon, Keith Gregory, John Pearson. Thermal modelling of an alternator for use in a prediction system. Proc. 2012 UKACC International Conference on Control. Cardiff, UK, 3-5 September 2012. pp. 455-460.

26. Boglietti A., Cavagnino A., Staton D., Shanel M., Mueller M., Mejuto C. Evolution and modern approaches for thermal analysis of electrical machines. IEEE Transactions on Industrial Electronics, 2009, vol. 56, Iss. 3, pp. 871-882.

27. Krok R. Electric mine motor thermal models aiding design and setting thermal protections Bulletin of the Polish Academy of Sciences. Technical Sciences, 2012, vol. 60, no. 1, pp. 103-110.

28. Lim C.H., Airoldi G., Bumby J.R., Dominy R.G., Ingram G.I., Mahkamov K., Brown N.L., Mebarki A., Shanel M. Experimental and CFD investigation of a lumped parameter thermal model of a single-sided, slotted axial flux generator. International Journal of Thermal Sciences, 2010, vol. 49, Iss. 9, pp. 1732-1741.

29. Ziuzev A.M., Metelkov V.P. Research of the start-up modes of multi-stage blower asynchronous drive. Proc. IEEE IX Int. Conf. on Power Drives Systems (ICPDS). Perm, October 3-7, 2016. pp. $1-5$.

30. Ziuzev A.M., Metelkov V.P. On an estimate of the rotor winding temperature at start-up of high-voltage induction motor. Proc. 2017 Int. Conf. on Industrial Engineering, Applications and Manufacturing (ICIEAM 2017). Saint-Petersburg, Russia, 16-19 May, 2017. pp. 1-6.

31. Younsi K., Neti P., Shah M., Zhou J., Krahn J., Weeber K., Whitefield C. On-line capacitance and dissipation factor monitoring of AC stator insulation. IEEE Transactions on Dielectrics and Electrical Insulation, 2010, vol. 17, Iss. 5, pp. 1441-1452.

32. Metelkov V.P., Borodin M.Yu., Kondakov K.A., Nesterov K.E. Using leakage currents to evaluate the induction motor insulation condition. Bulletin of the South Ural State University. Ser. Power Engineering, 2019, vol. 19, no. 1, pp. 67-74. In Rus.

33. Metelkov V.P., Ziuzev A.M., Chernykh I.V. System for estimating a residual resource of induction motor winding insulation based on capacitive leakage currents. Electrotechnical Systems and Complexes, 2019, no. 1 (42), pp. 53-58. In Rus.

Received: 23 December 2020.

\section{Information about the authors}

Anatoliy M. Ziuzev, Dr. Sc., professor, Ural Federal University.

Oleg V. Kryukov, Dr. Sc., Deputy Director, TSN-electro LLC.

Vladimir P. Metelkov, Dr. Sc., professor, Ural Federal University.

Sergey G. Mikhalchenko, Dr. Sc., Head of Industry Electronics Department, Tomsk State University of Control Systems and Radioelectronics. 